Abstract

\title{
Development of Targeted Therapies for Breast and Highly Aggressive Cancers ${ }^{\dagger}$
}

\section{Bulent Ozpolat}

Department of Experimental Therapeutics, Non-Coding RNA Center, Division of Cancer Medicine, The University of Texas MD Anderson Cancer Center, Houston, TX 77030, USA; bozpolat@mdanderson.org + Presented at the 3rd International conference on Natural Products for Cancer Prevention and Therapy, Kayseri, Turkey, 18-20 December 2019.

Published: 8 February 2020

\begin{abstract}
Cancer is one of the top two causes of deaths in the US and the world. Cancer therapy includes standard therapies such as surgery, chemotherapy and targeted therapies, by antibody and small molecule inhibitors, and immunotherapy (i.e., check point inhibitors, CAR-T cell therapy). More recently RNA-based targeted therapeutics were approved by FDA. Although there are about more than 100 targeted therapies, due to significant heterogeneity in patient tumors even in the same subtype of cancers, only faction of patient can benefit from targeted therapies due to lack of target expression in all patients. For some therapeutic targets, such as the most famous and one of the first identified oncogenes, KRAS, which is also considered as an "undrugabale" target expressed in cytoplasm and cannot be targeted by antibodies and small molecule inhibitors, currently there is no FDA approved inhibitors for patients. Therefore, gene targeted and RNA therapies overcome difficulties faced by the use of small molecule and antibodies provide promising new avenue for targeting these oncogenes. After several decades of research finally some of the gene targeted therapies emerged and are being tested in clinical trials. The talk will also give background in targeted therapies used in cancer patients and our novel targeted therapies. After overweening targeted therapies including gene therapies used in cancer with "pros of cons", my talk will focus on development of RNA, noncoding RNA, small molecule and natural compound-based targeted therapies on the several highly aggressive cancers including triple negative breast cancer, pancreatic, and lung cancer. We discovered and validated EF2 Kinase as a novel molecular target and showed their clinical significance by demonstrating their association with significantly shorter patient survival in TNBC. Using in vitro and in vivo tumor models in mice, we demonstrated that EF2-Kinase regulates, cell proliferation, invasion, and tumorigenesis and nanotherapeutics approaches for targeting EF2-Kinase is highly effective for treatment of patients with highly aggressive cancers.
\end{abstract}

Keywords: EF2-kinase; triple negative breast cancer; pancreatic cancer; ovarian cancer and lung cancer

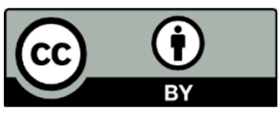

(c) 2020 by the authors. Licensee MDPI, Basel, Switzerland. This article is an open access article distributed under the terms and conditions of the Creative Commons Attribution (CC BY) license (http://creativecommons.org/licenses/by/4.0/). 